\title{
REESTRUCTURACIÓN ECONÓMICA Y TURISMO. LA NUEVA VERSIÓN DE LOS MOVIMIENTOS MIGRATORIOS EN LOS PAÍSES DE LA UNIÓN EUROPEA
}

\author{
Raúl LARDIÉS BOSQUE \\ Departamento de Geografía, King's College London \\ Universidad de Londres
}

\begin{abstract}
Resumen: En los países integrantes de la Unión Europea se han producido importantes cambios económicos, sociales y culturales, en el contexto de la reestructuración económica global. Todo ello ha traido consigo cambios importantes en el sector terciario, aumentando los contactos internacionales y la movilidad de la población.
\end{abstract}

En este artículo se analizan los nuevos tipos de movilidad intracomunitaria generados en el marco de esa internacionalización y reestructuración económica. En primer lugar se hace una revisión de los principales flujos migratorios, dirección, volumen y evolución entre los estados miembros de la Unión y, a continuación, un análisis de algunos de los principales tipos de movilidad desarrollados: movilidad académica, la de personal cualificado, la de población retirada, y el aumento de los desplazamientos por motivos turísticos a zonas del Mediterráneo.

Palabras clave: Migraciones internacionales, Unión Europea, reestructuración económica, internacionalización, migración internacional de población retirada, migración de personal cualificado, turismo.

\begin{abstract}
Important economic, social and cultural changes have emerged in the EU countries in the context of global economic restructuring. This restructuring has led to changes in tertiary sector of the European economy, the increase of international exchanges, and the socio-spatial mobility of the population.

The aim of this paper is to examine new types of mobility between EU countries generated within the setting of internationalization and economic restructuring. First, the flow, direction, volume and evolution of international migration is reviewed. Second, migration development; academic mobility, skilled international migration, international retirement migration, and the increase of the movements due to tourist motivations to Mediterranean areas are analysed.
\end{abstract}

Key words: International migration, European Union, economic restructuring, internationalization, international retirement migration, skilled migration, tourism. 


\section{INTRODUCCIÓN}

En las últimas dos y tres décadas se han producido importantes transformaciones en el conjunto europeo, tanto de orden económico, político o institucional. Esos cambios, asociados fundamentalmente a la construcción de la Unión Europea, han contribuido directamente al redibujamiento del mapa económico europeo, y han tenido consecuencias espaciales importantes en la distribución y en la movilidad de la población. Paralelamente a esos cambios se ha desarrollado todo un complejo sistema de migraciones en el cual todo tipo de desplazamientos tienen cabida: la movilidad tanto interna como externa, intra y extra comunitaria materializada en múltiples movimientos como los temporales o estaciones, de baja o alta cualificación, los de retornados a sus países de origen, en busca de mejor situación laboral o de sol en la jubilación, etc. Toda esa variedad de desplazamientos se entremezclan y solapan con la atracción continua que países de fuera del entorno europeo siguen ejerciendo para la población europea; pero, a la vez, son el reflejo de las múltiples y variadas relaciones económicas y humanas que se dan en el espacio europeo, además de un vector hacia la integración y la construcción de un espacio europeo común y sin fronteras.

A raíz de la entrada en vigor del Tratado de Roma (1968) y del acuerdo de Schengen firmado hasta 1993 por nueve estados comunitarios no se han producido aumentos en las migraciones en las últimas décadas. Pese a las medidas que el Tratado introdujo, las estadísticas confirman que el volumen total de migraciones entre los estados miembros ha declinado durante la década de los 80. Sin embargo, la nueva situación económica, social y cultural europea está propiciando el desarrollo de nuevas formas de movilidad e intercambios entre los países comunitarios, siendo muchas veces estadísticamente invisibles. Cuando hablamos de migraciones hay que hacer referencia a la gran diversidad de ellas, formas y contextos en los que se producen, por lo que no todas ellas tienen una explicación común. El móvil económico fue el principal en los desplazamientos masivos de las pasadas décadas, cuando miles de trabajadores de países del sur europeo se establecieron en los países del norte. Hoy en día, los argumentos económicos ya no intervienen en el proceso migratorio con la misma fuerza que lo hicieron en el pasado, sino que muchos de los movimientos ahora están más ligados a otras razones, en parte debido a la desaparición de las diferencias económicas entre los países comunitarios. A eso hay que añadir el desarrollo de las modernas redes de transporte y comunicaciones, que hacen que se modifique el concepto físico y la percepción las distancias.

En este artículo nos proponemos llevar a cabo un análisis e interpretación. de algunas de las migraciones que se están produciendo, infuenciadas, directa o indirectamente, por el proceso de reestructuración económica y el desarrollo de determinados sectores económicos. Tras hacer una revisión general a nivel europeo de los flujos migratorios, dirección, volumen, y evolución de los mismos, en primer lugar 
analizaremos la movilidad académica y la importancia de los desplazamientos del personal investigador y del mundo de la educación como un efecto más de la reestructuración y la internacionalización de la economía. En segundo lugar haremos referencia a uno de los más claros exponentes de esa reestructuración, a nivel de las empresas, como está siendo la movilidad del personal cualificado. Y en tercer y cuarto lugar, de una forma conexa, nos ocuparemos de cómo determinados factores no económicos y sociales, fundamentalmente, unidos al desarrollo del turismo en zonas del Mediterráneo, están favoreciendo la movilidad de determinados grupos de emigrantes a los países del sur de Europa.

\section{LA GEOGRAFÍA TRADICIONAL DE LAS MIGRACIONES INTRACOMU- NITARIAS: DE LA PERIFERIA AL CENTRO}

Europa en su conjunto, pero en particular los países que hoy constituyen la Unión Europea, han sido un foco tradicional de inmigración desde otras partes del mundo; sin embargo, la movilidad intra-europea ha sido menos numerosa. Durante muchas décadas, esos movimientos internos de población han sido relativamente escasos en comparación con los flujos desde el Este al Oeste de Europa, y sobre todo del Magreb y ciertos países latinoamericanos y asiáticos a los países mediterráneos (CHAMPION, 1995).

El análisis de la población comunitaria durante la década de los 80 advierte el claro crecimiento de la población en estos países, producido, en parte, por aumento de los inmigrantes de fuera del espacio comunitario. Si en 1993 había 16.879.200 inmigrantes en los 15 países que hoy constituyen la U.E., tan sólo 5.291 .838 eran originarios de países de la misma U.E. Esa cifra supone un porcentaje de comunitarios del $31,35 \%$, mientras que los procedentes de otros continentes sobrepasan el $50 \%$ (EUROSTAT, 1995). Si hace dos décadas los principales flujos de inmigración tenían su origen en los países del sur de Europa con dirección a los industrializados del norte, hoy en día provienen del exterior (sólo Turquía aporta un $15,24 \%$ de la inmigración externa a la U.E., Africa un 17,71\%, América un 5,61\% y Asia un 11\%). Frente a ese masivo aumento de población no comunitaria, sólo el $1,43 \%$ de toda la población comunitaria vivía en 1993 en un país comunitario diferente al suyo, lo cual supone menos de 5,3 millones de personas de un total de casi 369 millones (Tabla 1). Esa es una cifra, también, relativamente baja si la comparamos con la movilidad extracomunitaria ya que el número de comunitarios residiendo fuera de la Unión Europa es tres veces superior; es decir, 15 millones de personas (EUROSTAT, 1995). 
Tabla 1.- Emigrantes de cada país comunitario en otros países de la U.E., 1993.

\begin{tabular}{lrrrr}
\hline País de origen & $N^{0}$ Emigrantes & \% Emigr. (1) & Población total & \% Emigr,/Pobl. (2) \\
\hline Italia & 1.155 .636 & 21,84 & 56.960 .300 & 2,0 \\
Portugal & 829.902 & 15,68 & 9.864 .600 & 8,41 \\
Irlanda & 498.051 & 9,41 & 3.563 .300 & 13,98 \\
España & 478.481 & 9,01 & 39.048 .000 & 1,22 \\
Grecia & 418.701 & 7,91 & 10.350 .300 & 4,04 \\
Reino Unido & 365.011 & 6,90 & 57.222 .000 & 0,64 \\
Alemanania & 352.520 & 6,66 & 80.974 .600 & 0,43 \\
Francia & 305.648 & 5,77 & 56.652 .000 & 0,54 \\
Países Bajos & 254.194 & 4,80 & 15.239 .200 & 1,67 \\
Austria & 216.055 & 4,08 & 7.795 .800 & 2,77 \\
Finlandia & 143.440 & 2,71 & 5.055 .000 & 2,84 \\
Bélgica & 124.083 & 2,34 & 10.068 .300 & 1,23 \\
Dinamarca & 72.020 & 1,36 & 5.180 .600 & 1,39 \\
Suecia & 64.049 & 1,21 & 8.692 .000 & 0,74 \\
Luxemburgo & 14.047 & 0,26 & 395.200 & 3,55 \\
Total U.E. & 5.291 .838 & $100 \%$ & 368.977 .500 & \\
\hline
\end{tabular}

(1) Porcentaje de Emigrantes en cada país respecto al total de la U.E. (15 países).

(2) Porcentaje de Emigrantes en cada país respecto al Total de su población.

Fuente: EUROSTAT (1995). Elaboración propia.

El análisis del volumen y dirección de estos flujos entre los países de la Unión (mediante el número de residentes) muestra que el 54,44\% del total de los emigrantes tiene su origen en países mediterráneos como Italia $(21,84 \%)$, Portugal $(15,68 \%)$, España $(9,01 \%)$ y Grecia $(7,91 \%)$, generando entre los cuatro países más de la mitad de las migraciones internas entre los quince países comunitarios. Por contra, los emigrantes con origen en países del norte es mucho menor (Tabla 1). El impacto que estos emigrantes tienen en sus respectivos países, en relación con la población total de cada uno de ellos, es muy desigual. Fn países como Italia, con el mayor número de emigrantes, ese número de emigrantes representa un escaso porcentaje en relación a su población total (sólo un 2\%) (Tabla 1). Por un lado, en países tradicionalmente emigratorios como Irlanda y Portugal es en los que mayor impacto ha tenido la salida 
de estos emigrantes en relación a sus poblaciones totales $(13,98 \%$ y $8,41 \%$ respectivamente). Por otro, los países tradicionalmente receptores ( $\mathrm{y}$ a la vez menos expulsores) son en los que menos ha supuesto esta pérdida de población; es el caso del Reino Unido, Alemania y Francia, donde ese porcentaje está entre el 0,4 y 0,6\%. En España, aunque el número de personas que residen en otros países comunitarios representa una cifra considerable (478.481), respecto al total de la población es poco más del 1,2\%. En relación a este análisis de los residentes comunitarios en otros países de la Unión, queda claro que la geografía de las migraciones internas comunitarias está caracterizada por una fuerte polarización, determinando una dirección sur-norte en esas corrientes, debida en parte a los movimientos producidos en las pasadas décadas.

Si esos son los principales países de origen de las migraciones, los receptores, por contra, no son muy numerosos. Alemania (1.717.177 de inmigrantes comunitarios recibidos, que representan el 2,12\% de su población), Francia (con 1.315.438, y el $2,32 \%$ ), Reino Unido (con 770.000, y el 1,35\%) y Bélgica (con 541.613, y e1 5,38\%) son los principales de acogida (Tabla 2). En esa misma tabla, una simple comparación entre los porcentajes de inmigrantes según las nacionalidades para esos cuatro países fundamentalmente receptores permite obtener aigunas conclusiones. Una de ellas es que, en casi todos ellos, los dos grupos más numerosos suelen proceder de países del sur (Italia, Portugal o Grecia). Por el contrario, si nos fijamos en los países mediterráneos, suelen ser los residentes comunitarios procedentes del norte los que constituyen los grupos más numerosos: entre ellos los británicos en primer lugar, seguidos de los alemanes, y despúes de los franceses. Hay que tener en cuenta la excepción de países como Finlandia o Suecia, cuya atracción por países más próximos como Alemania o Dinamarca hace evitar generalmente su presencia en los más meridionales. Salvo pocas excepciones, la generalidad es que los países del sur constituyen destinos importantes para el establecimiento de los inmigrantes del norte. Aunque a eso se debe hacer una matización, y es que en algunos países como Alemania, Países Bajos o Francia, en cifras absolutas residen más ciudadanos procedentes del norte, que en los países del sur. Es el caso de los alemanes, cuyo grupo más numeroso está en Austria (57.300), seguido de Francia (52.723) y Reino Unido (51.000); o de los británicos, cuyo número más alto se concentra en Alemania (107.130), aunque seguido ya de España (53.441), Francía (50.422) y los Países Bajos (44.117) (Tabla 2).

A la vista de todo esto, los intercambios comunitarios permiten discernir tres tipos diferentes de situaciones migratorias, en el interior de los países de la U.E.

1) Los países tradicionalmente migratorios (Irlanda, Italia, Portugal, Grecia y España), con una balanza migratoria negativa. Como un país emigratorio, pero por razones distintas a los anteriores, aparece también Finlandia, con 143.440 residentes en otros paises comunitarios (el $77,71 \%$ de ellos en Suecia) (Tabla 1), mientras que se 
contabilizan tan sólo 12.239 inmigrantes (el 52,75\% de ellos procedentes de Suecia) (Tabla 2).

2) Países mayoritariamente receptores como Luxemburgo ${ }^{1}$, Alemania, Francia, Reino Unido y Bélgica. Suecia.

3) Países con intercambios equilibrados, como los Países Bajos, Dinamarca o

Sin embargo, este análisis de los residentes en otros países de la U.E. no refleja fielmente todos los movimientos de población que se están produciendo actualmente en Europa, sino que responde más bien a las masivas migraciones producidas hace dos y tres décadas del sur al norte europeo. Se trata de un análisis de alguna manera "estático", en el sentido de que ese número de residentes constituye una herencia, y está ligado a la historia de las migraciones europeas. La actual "escena" migratoria comunitaria, complicada y multifactorial como hemos señalado, no queda suficientemente reflejada en este análisis estadístico.

Las transformaciones económicas, políticas, sociales o institucionales asociadas a la construcción de la Unión Europea har contribuido al surgimiento de nuevas fuerzas económicas con consecuencias espaciales importantes en la distribución y en la movilidad de la población. El mapa tradicional con las migraciones en sentido surnorte está cambiando, y nuevos flujos, mucho más variados, y provocados por causas distintas, se están produciendo. Veamos a continuación algunos de los cambios producidos, y su efecto en la movilidad.

\section{REESTRUCTURACIÓN ECONÓMICA, DESCENTRALIZACIÓN URBANA Y DESARROLLO DE NUEVAS FORMAS DE MOVILIDAD}

Antes de prestar atención a los nuevos tipos de movilidad que se están produciendo en Europa, conviene contextualizarlos, refiriéndonos a un grupo de factores que proporcionan una interpretación de los más recientes cambios espaciales producidos a escala urbana y regional, todo ello en el marco de la reestructuración económica.

\footnotetext{
1 Aunque en la Tabla 2 no se incluye Luxemburgo, en 1987 era el país comunitario que, proporcionalmente a su población, tenía el más alto porcentaje de inmigrantes comunitarios (88.600), lo que suponía un $24,2 \%$ de su población total (SIMON, 1992).
} 
Tabla 2. Residentes de países comunitarios en los países de la U.E., 1993. (1)

\begin{tabular}{|c|c|c|c|c|c|c|c|c|c|c|c|c|}
\hline \multirow[b]{3}{*}{ País de origen } & \multicolumn{10}{|c|}{ PAISES DE RECEPCIÓN } & & \\
\hline & \multicolumn{2}{|c|}{ BÉLGICA } & \multicolumn{2}{|c|}{ DINAMARCA } & \multicolumn{2}{|c|}{ ALEMANIA } & \multicolumn{2}{|c|}{ GRECIA } & \multicolumn{2}{|c|}{ ESPAÑA } & \multicolumn{2}{|c|}{ FRANCIA } \\
\hline & $N^{n}$ & $\%$ & $\mathrm{~N}^{\mathrm{n}}$ & $\%$ & $N^{n}$ & $\%$ & $N^{10}$ & $\%$ & $N^{\prime \prime}$ & $\%$ & $\mathrm{~N}^{3}$ & $\%$ \\
\hline Bélgica & --- & --- & 357 & 0,9 & 21.986 & 1,3 & 1.667 & 2,6 & 7.221 & 4,0 & 56.129 & 4,3 \\
\hline Dinamarca & 2.783 & 0,5 & -.-- & $-\ldots$ & 18.252 & 1,1 & 1.636 & 2,5 & 3.984 & 2,2 & 3.544 & 0,3 \\
\hline Alemania & 29.318 & 5,4 & 8.896 & 21,9 & ---- & $\cdots$ & 14.079 & 21,8 & 30.493 & 16,8 & 52.723 & 4,0 \\
\hline Grecia & 19.987 & 3,7 & 581 & 1,4 & 343.902 & 20,0 & --- & -.-- & 474 & 2,3 & --- & .... \\
\hline España & 49.459 & 9,1 & 1.009 & 2,5 & 133.847 & 7,8 & 1.034 & 1,6 & $-\ldots--$ & --- & 216.047 & 16,4 \\
\hline Francia & 95.165 & 17,6 & 2.228 & 5,5 & 90.877 & 5,3 & 7.993 & 12,4 & 22.644 & 12,5 & $\ldots$ & -... \\
\hline Irlanda & 2.764 & 0,5 & 961 & 2,3 & 13.761 & 0.8 & 709 & 1,1 & 2.055 & 1,1 & 3.542 & 0,3 \\
\hline Italia & 217.534 & 40,2 & 2.004 & 4,9 & 557.709 & 32,5 & 7.378 & 11,4 & 13.580 & 7,7 & 252.759 & 19,2 \\
\hline Luxemburgo & 4.585 & 0,8 & 13 & 0,0 & 5.405 & 0,3 & 47 & 0,1 & 111 & 0,1 & 3.040 & 0,2 \\
\hline Países Bajos & 69.712 & 12,9 & 2.268 & 5,6 & 113.552 & 6,7 & 3.693 & 5,7 & 10.499 & 5,8 & 17.881 & 1,4 \\
\hline Austria & 1.071 & 0,2 & 590 & 1,5 & 185.278 & 10,8 & 1.858 & 2,9 & 1.469 & 0,9 & 3.280 & 0,2 \\
\hline Portugal & 20.495 & 3,8 & 360 & 0,9 & 98.918 & 5,8 & 411 & 0,6 & 28.631 & 15,8 & 649.714 & 49,4 \\
\hline Finlandia & 791 & 0,1 & 1.912 & 4,7 & 12.173 & 0,7 & 1.155 & 1,8 & 1.944 & 1,1 & 1.552 & 0,1 \\
\hline Suecia & 3.083 & 0,6 & 8.430 & 20,8 & 14.387 & 0,8 & 2.296 & 3,5 & 5.258 & 2,9 & 4.805 & 0,4 \\
\hline Reino Unido & 24.866 & 4,6 & 10.920 & 26,9 & 107.130 & 6,2 & 20.705 & 32,0 & 53.441 & 29,4 & 50.422 & 3,8 \\
\hline TOTAL UE & 541.613 & 100 & 40.529 & 100 & 1.717.177 & 100 & 64.661 & 100 & 181.804 & 100 & 1.315 .438 & 100 \\
\hline$\%$ UE / Pobl. & 2) 5,38 & & 0,78 & & 2,12 & & 0,62 & & 0,47 & & 2,32 & \\
\hline
\end{tabular}




\begin{tabular}{|c|c|c|c|c|c|c|c|c|c|c|c|c|c|c|}
\hline \multirow[b]{3}{*}{ País de origen } & \multicolumn{14}{|c|}{ PAÍSES DE RECEPCIÓN } \\
\hline & \multicolumn{2}{|c|}{ ITALIA } & \multicolumn{2}{|c|}{ РP.BB. } & \multicolumn{2}{|c|}{ AUSTRIA. } & \multicolumn{2}{|c|}{ PORTUG. } & \multicolumn{2}{|c|}{ FINLAND. } & \multicolumn{2}{|c|}{ SUECIA. } & \multicolumn{2}{|c|}{ R. UNIDO } \\
\hline & $N^{\prime \prime}$ & $\%$ & $\mathrm{~N}^{\prime \prime}$ & $\%$ & $N^{\prime \prime}$.. & $\%$ & $N^{\prime \prime}$ & $\%$ & $N^{\prime \prime}$ & $\%$ & $N^{\prime \prime}$ & $\%$ & $N^{i \prime}$ & $\%$ \\
\hline Bélgica & 4.636 & 2,9 & 24.023 & 12,7 & 500 & 0,6 & 1.118 & 3,4 & 67 & 0,5 & 379 & 0,2 & 6.000 & 0,8 \\
\hline Dinamarca & 2.416 & 1,5 & 1.856 & 1,0 & 400 & 0,5 & 499 & 1,5 & 474 & 3,9 & 27.176 & 14,5 & 9.000 & 1,2 \\
\hline Alemania & 39.456 & 24,6 & 49.333 & 26,1 & 57.300 & 72,3 & 5.404 & 16,5 & 1.588 & 13,0 & 12.930 & 6,9 & 51.000 & 6,6 \\
\hline Grecia & 16.243 & 10,1 & 5.554 & 3,0 & 1.000 & 1,3 & 65 & 0,2 & 229 & 1,9 & 5.666 & 3,0 & 25.000 & 3,2 \\
\hline España & 15.559 & 9,7 & 16.788 & 8,9 & 700 & 0,9 & 7.734 & 23,7 & 316 & 2,6 & 2.988 & 1,6 & 33.000 & 4,3 \\
\hline Francia & 25.381 & 15,8 & 9.970 & 5,3 & 2.200 & 2,8 & 3.674 & 11,2 & 393 & 3,2 & 3.123 & 1,7 & 42.000 & 5,4 \\
\hline Irlanda & 2.530 & 1,6 & 4.441 & 2,3 & 200 & 0,2 & 244 & 0,7 & 103 & 0,8 & 741 & 0,4 & 466.000 & 60,5 \\
\hline Italia & --- & $\ldots$ & 17.284 & 9,1 & 8.600 & 10,8 & 1.353 & 4,1 & 448 & 3,7 & 3.987 & 2,1 & 73.000 & 9,5 \\
\hline Luxemburgo & 190 & 0,1 & 298 & 0,2 & 300 & 0,4 & 36 & 0,1 & 5 & 0,0 & 17 & 0,0 & $\ldots$ & --- \\
\hline Países Bajos & 7.032 & 4,4 & -.-- & --- & 2.600 & 3,3 & 2.010 & 6,1 & 338 & 2,8 & 2.609 & 1,4 & 22.000 & 2,9 \\
\hline Austria & 8.322 & 5,2 & 2.996 & 1,6 & $\ldots$ & $\cdots$ & 302 & 0,9 & 151 & 1,2 & 3.738 & 2,0 & 7.000 & 0,9 \\
\hline Portugal & 5.285 & 3,3 & 9.352 & 4,9 & 200 & 0,2 & $\cdots$ & $-\cdots$ & 71 & 0,6 & 1.465 & 0,8 & 15.000 & 1,9 \\
\hline Finlandia & 1.638 & 1,0 & 1.053 & 0,6 & 500 & 0,6 & 245 & 0,7 & $\ldots$ & -- & 111.477 & 59,6 & 9.000 & 1,2 \\
\hline Suecia & 3.242 & 2,0 & 1.970 & 1,0 & 1.400 & 1,8 & 722 & 2,2 & 6.456 & 52,7 & $\ldots$ & $\cdots$ & 12.000 & 1,6 \\
\hline Reino Unido & 28.390 & 17,7 & 44.117 & 23,3 & 3.400 & 4,3 & 9.284 & 28,4 & 1.600 & 13,1 & 10.736 & 5,7 & $\ldots$ & -.- \\
\hline TOTAL UE & 160.320 & 100 & 189.035 & 100 & 79.300 & 100 & 32.630 & 100 & 12.239 & 100 & 187.032 & 100 & 770.000 & 100 \\
\hline$\%$ UE/ Pobl. (2) & 0,28 & & 1,24 & & 1,02 & & 0,33 & & 0,24 & & 2,15 & & 1,35 & \\
\hline
\end{tabular}

(1) Excluidos Irlanda y Luxemburgo por falta de datos desagregados a nivel nacional.

(2) Porcentaje de comunitarios en cada país respecto al total de la población.

Fuente: EUROSTAT (1995). Elaboración propia. 
En un intento por explicar el proceso de concentración-desconcentración de la población europea y los cambios migratorios producidos en relación con el fenómeno metropolitano, autores como CHAMPION e ILLERIS (1990) apuntan a la existencia de ciertos factores que influyen en la distribución de la población y de los trabajos en el marco de los cambios económicos y urbanos que se están produciendo en la nueva Europa.

Un factor importante que ha influido en la última década en las variaciones de la movilidad de la población ha sido la contraurbanización, con las implicaciones que está teniendo en la concentración y desconcentración de la población (CHAMPION, 1995). El proceso de contraurbanización ha alterado el esquema migratorio tradicional desde las regiones periféricas hacia los centros industriales y urbanos europeos. Sin embargo, muchos son los factores que influyen en el proceso de descentralización urbana, y que tienen influencia en la nueva redistribución y movilidad de la población. Este crecimiento de población en zonas tradicionalmente periféricas y/o rurales, se ha debido a varios factores entre los que destacan la crisis energética del 73 que conllevó la de muchas actividades industriales, además de otros como la expansión de 1 a educación o la modernización de las infraestructuras públicas y privadas en zonas periféricas o rurales. Junto a ello no hay que olvidar los intereses por parte de muchos gobiernos nacionales por las políticas de regeneración de los espacios urbanos, lo que ha supuesto el vaciamiento de muchos de esos espacios congestionados, respondiendo así a muchos problemas ocasionados por la concentración urbana.

Pero entre esos factores hay que destacar las importantes fuerzas centrífugas que conducen a la desconcentración de población y trabajos a escalafones menores en la jerarquía urbana. Ello implica nuevas pautas de localización de determinados servicios a las empresas y de muchas oficinas centrales de empresas. Esas nuevas pautas demandan tanto altos niveles de accesibilidad física como mano de obra de gran cualificación, además de nuevos enclaves urbanos/metropolitanos de menor jerarquía con áreas de trabajo menos congestionadas. Por otro lado, la atracción de los espacios menos congestionados reorienta la actividad económica hacia regiones periféricas y conduce a la instalación de plantas industriales en zonas de suelo y mano de obra más barata. Este es un proceso que no sólo afecta al sector industrial en las tareas de produccioón, sino también en las de decisión con la instalación de oficinas y centros de decisión. Finalmente, las mejoras en los transportes y las comunicaciones, junto con la expansión del turismo y el ocio, son factores que están favoreciendo también el proceso de desconcentración urbana y la búsqueda de espacios rurales para el establecimiento de primeras y segundas residencias. Ese es un proceso detectado tanto a escala nacional, como internacional, como luego veremos, con desplazamientos de población desde países del norte de Europa a los del sur, con importantes transformaciones espaciales (CHAMPION, 1995). 
Atendiendo a factores de tipo únicamente demográfico (envejecimiento de la población europea o la reducción de las tasas de fertilidad en los países del sur) o de indole socioeconómico como la reducción de las diferencias económicas y sociales entre los países del norte y del sur, sería demasiado simple intentar explicar un fenómeno tan complejo como es el de las migraciones intracomunitarias. La nueva libertad en la movilidad de la población ha creado una nueva situación, acompañada de un nuevo escenario económico en estos países, y de un nuevo contexto sociológico creado por el funcionamiento de un mercado único; una situación que, sin duda, potencia nuevos movimientos de población hasta ahora inexistentes como los de estudiantes y gente joven, profesionales de alta cualificación, $y$ otros muchos ligados directa o indirectamente con el turismo en zonas del Mediterráneo. No se trata de movimientos masivos como los producidos en las pasadas décadas. $Y$ de hecho, escapan frecuentemente a las estadísticas debido, en parte, a que la mayoría de ellos son de corta duración.

\section{a) Internacionalización de la educación y movilidad académica}

La internacionalización de las actividades educativas y de investigación se ha convertido en un hecho evidente, y de gran impacto tanto económico como social. En términos generales, se puede decir que la globalización en la economía ha conducido a la expansión del número de prácticas y de intercambios entre universidades y otros centros de educación e investigación, tanto a nivel de pre-graduados como de postgraduados, y de personal docente e investigador. Pero para entender esta nueva dimensión internacional de la educación debemos recurrir a dos tipos de factores, unos externos, y otros internos (KAMEOKA, 1996). (i) Consideraciones externas apuntan a que los cambios actuales en el mercado de trabajo demandan continuamente personal más preparado y especializado, lo cual se asocia al conocimiento de otros métodos, técnicas y ambientes de trabajo. En el caso de los países de la Unión Europea, el especial interés por una integración en términos educativos y académicos, ha conducido a la puesta en marcha de varios programas comunitarios de ámbito internacional, favorecedores de la movilidad de estudiantes entre los países miembros de la Unión.

(ii) Esa misma globalización e internacionalización nos lleva a la consideración de los otros factores, los internos, o a las respuestas que la mundialización e internacionalización de los acontecimientos ha tenido en las instituciones. Cada vez más, existe gran relación entre la internacionalización curricular y la movilidad de estudiantes y del personal académico e investigador. A nivel de acuerdos entre instituciones particulares, cada vez son más numerosos los intercambios entre centros, también, tanto entre estudiantes como entre personal investigador y docente. A finales de los años 80 , tan sólo el $2 \%$ de los estudiantes habían vivido en otro país comunitario distinto al suyo. Francia y la República Federal de Alemania fueron en su comienzo los países de destino de los principales estudiantes comunitarios en intercambio, e Italia y 
también la R.F. de Alemania los principales países de salida. Entre los programas comunitarios puestos en marcha, quizá haya sido el programa ERASMUS (actualmente el programa SOCRATES engloba a los antiguos Erasmus, Lingua, Eurydice y Arion) el que mayor movilidad haya generado. Este programa fue concebido para favorecer los intercambios de corta duración (de tres meses a un año) entre los estudiantes de países miembros, e inicialmente intentó que el 10\% de los estudiantes comunitarios tuvieran al menos, una experiencia de estudios en el extranjero. En el curso académico 1993/94 este programa movilizó a 1.231 estudiantes incluyendo a 98 instituciones, y su cuantía total para el quinquenio 1995-1999 es de 850 millones de ECUs. Junto a él, otros programs importantes son YES o TEMPUS.

Por otro lado, en estos momentos está vigente en la Unión Europea el Cuarto Programa Marco comunitario (Fourth Framework Programme, 1994-1998), cuyo objetivo principal es "el desarrollo y mejor utilización de los recursos humanos en la Comunidad mediante la formación y la movilidad de los investigadores" (EUROPEAN COMMISSION, 1996). Las becas destinadas a este cuarto programa cuentan para todo el cuatrienio con 394 millones de ECUs que se dedicarán a la investigación y al intercambio de investigadores, y en cuyo periodo se prevé la movilidad de más de 5.000 personas (número de becas concedias) para la formación de investigadores en otros países comunitarios. Estamos hablando de una movilidad no masiva, pero de gran impacto. Sin embargo, geográficamente conviene hacer una matización, y es que la mayoría del personal desplazado procede de países mediterráneos del sur, y con destino a los del norte. Sirva de ejemplo que en 1995 el mayor número de becas concedidas por la U.E. (predoctorales y postdoctorales) se otorgó a investigadores españoles (PÉREZ, 1996).

Finalmente, destacar también los esfuerzos que las instituciones privadas de los países comunitarios están haciendo para la potenciación de esta movilidad, y sin olvidar otros, a escala nacional, con estrategias incluso más de carácter político como la de "coopération civil à l' étranger, en Francia. Como vemos, estamos hablando de una movilidad cuyo aumento parece claro, en la medida en que los diversos esfuerzos que se están realizando favorecen este tipo de intercambios de corta y media duración, y también en la medida en que estos intercambios temporales suelen implicar generalmente a gente joven (más independiente desde el punto de vista familiar), lo cual facilita mucho más la movilidad.

b) Internacionalización de las empresas y movilidad del personal cualificado

Otro tipo de migración relacionada con la reestructuración de la economía europea y con la internacionalización de las empresas y sus cambios organizativos es la de trabajadores cualificados. Esta migración forma parte de un nuevo fenónemo que se está viviendo en los países de la Unión Europa, y que implica el desplazamiento de 
personal cualificado, como directivos y ejecutivos de empresas, científicos, u otros trabajadores especializados y/o de alta cualificación, casi siempre en busca de mejoras profesionales (FINDLAY, 1995). En un proceso de mayor especialización y creación de un sistema jerárquico en las funciones y tareas de control, las compañías tienen que responder rápidamente a las nuevas situaciones de desarrollo y cambio, lo cual conlleva la mayoría de las veces una organización más global e internacional (SALT, 1992). Ese sistema jerárquico implica una división espacial con la separación física de las funciones de control y las de producción, de forma que las primeras se tienden a concentrar cada vez más en áreas metropolitanas de ciudades de tamaño medio (antes fundamentalmente en las grandes). Por contra, las fábricas son relegadas a localizaciones periféricas donde la mano de obra es más barata y los incentivos para la producción son mayores. La consecuencia de este proceso es que con esta nueva organización geográfica se generan nuevos desplazamientos entre los centros de decisión y producción, que está en la base de la movilidád del personal cualificado.

El hecho de que estemos ante un fenómeno relativamente reciente, y también ante un tipo de movilidad muy específica y escasa, hace que exista escasa información disponible tanto sobre el volumen y naturaleza como sobre las características de estas migraciones. Como ya hemos indicado, ello es debido, en parte, a que en una Europea donde cada vez es más factible la libre circulación de personas y trabajadores, este grupo de emigrantes, como otros muchos, escapan a las estadísticas por lo cual es prácticamente imposible conocer su número exacto. Además, la pertenencia a clases medias, acomodadas, junto con su cultura y color, convierte a estos migrantes en socialmente "invisibles" (SALT, 1992).

Desde el punto de vista geográfico, uno de los efectos que ha tenido la reestructuración económica ha sido la variación de la naturaleza y la expresión espacial del "corazón" de la economía europea. Geográficamente, ese corazón se ha extendido desde las zonas centrales del Rhin y del Ruhr hacia regiones más periféricas como Cataluña, el sureste francés, y norte y centro de Italia. Las presiones existentes, por tanto, en el actual mercado de trabajo estimulan el desarollo de esta "migración sofisticada", como la ha denominado G. SIMON (1993), tanto a las regiones centrales de la economía europea como a otras en expansión en el sur de Europa con ciudades como Barcelona, Toulouse, Montpellier, Génova o Milán como cabeceras principales de esta "red mediterránea". De esta forma, la nueva reespacialización económica europea está ofreciendo nuevas oportunidades a ciertos grupos de emigrantes para su desplazamiento a regiones "periféricas" (KING, 1997).

En general, el avance tecnológico y el desarrollo de las actividades terciarias suponen un aumento en la demanda de profesionales cada vez más cualificados. En algunos países comunitarios hay una falta notable de ingenieros y de personal directivo cualificado. Es precisamente en ese estrato profesional donde más puestos de 
trabajo se están creando en la actualidad, y uno de los de mayor demanda de empleo en muchos países europeos. Los Países Bajos son un claro ejemplo de la importancia de los trabajadores de alta cualificación. P. MUUS (1991) analiza la evolución del número de trabajadores extranjeros en el país en tres ramas del sector terciario (científicos, directivos y ejecutivos), de 1983 a 1989. En las tres categorías aumentó el número de extranjeros ocupados, pasando de 43.000 en 1983 a 60.000 en 1989, lo cual supone un incremento del $40 \%$ comparado con el del 10\% registrado en el total de empleos ocupados por los nacionales. Alrededor de dos tercios de todos esos trabajadores procedían de algún país comunitario, lo cual no deja de ser una proporción baja respecto a los muchos trabajadores que acudían al país hace dos y tres décadas procedentes de otros países europeos.

En el caso de Alemania, aunque el número total de trabajadores extranjeros (comunitarios y no comunitarios) disminuyó entre 1977 y 1989, la participación de trabajadores cualificados sobre el total aumentó considerablemente (WERNER, 1991). En Finlandia, aproximadamente el 30\% de los trabajadores extranjeros está por encima de la categoría de trabajadores "de cuello blanco", estando sobrerepresentados en determinadas ramas como la educación y la investigación. Así, franceses e británicos predominan en educación trabajando como profesores de idiomas, alemanes y suizos en puestos directivos de empresas y en el comercio, mientras que los procedentes de países europeos del sur se concentran en categorías profesionales más bajas y peor pagadas (MAJAVA y PENTTINEN, 1991). Otro de los países que está recibiendo mucho personal cualificado de otros vecinos comunitarios es el Reino Unido. Según las fuentes consultadas por J. SALT (1992), un 36,9\% del total de los inmigrantes con empleo en 1989 se incluían en las categorías de trabajadores con carrera y directivos (professional and managerial). Salt destaca que entre los inmigrantes, hay una tendencia por parte de los procedentes de países comunitarios a trabajar en puestos cualificados, mucho mayor que entre los propios británicos; y entre los hombres más que entre las mujeres. Entre el total de los inmigrantes "cualificados" supone una mayoría (44\%) los que llegan por traslado de alguna empresa, pero cuando se incluyen los procedentes de países de la U.E., ese porcentaje todavía aumenta más.

En la Tabla 3 se establece una evolución del empleo anual entre 1983 y 1991 para extranjeros y nacionales en seis países comunitarios, y en dos ramas de la economía del sector terciario. De las dos ramas, la primera, que es la que mejor engloba al personal cualificado del que estamos hablando, demuestra que, anualmente, el porcentaje de crecimiento de los trabajadores extranjeros ocupados en esas actividades ha sido mayor que el crecimiento de los trabajadores nacionales. 
Tabla 3.- Crecimiento anual comparado de los empleos totales y de los empleos para extranjeros por ramas económicas en algunos países de la U.E., 1983-1991 (en porcentajes).

\begin{tabular}{lccccccccccccc}
\hline & \multicolumn{2}{c}{ BÉLGICA } & \multicolumn{2}{c}{ ALEMANIA } & \multicolumn{2}{c}{ FRANCIA } & \multicolumn{2}{c}{ LUXEMB. } & \multicolumn{2}{c}{ PP.BAJOS } & \multicolumn{3}{c}{ R. UNIDO } \\
& $\mathrm{N}(1)$ & $\mathrm{E}(2)$ & $\mathrm{N}$ & $\mathrm{E}$ & $\mathrm{N}$ & $\mathrm{E}$ & $\mathrm{N}$ & $\mathrm{E}$ & $\mathrm{N}$ & $\mathrm{E}$ & $\mathrm{N}$ & $\mathrm{E}$ \\
\hline Rama 1 & 3,6 & 4,1 & 3,9 & 7,1 & 3,6 & 2,8 & 6,1 & 10,2 & 7,3 & 9,0 & 6,3 & 7,9 \\
Rama 2 & 1,6 & 0,8 & 0,7 & 0,9 & 1,4 & $-4,5$ & 1,9 & 9,1 & 1,6 & 22,4 & 2,4 & 4,8 \\
\hline
\end{tabular}

Rama 1: Bancos, finanzas, seguros y servicios a las empresas.

Rama 2: Administración pública, defensa nacional, seguridad social, representación diplomática, organizaciones internacionales y fuerzas armadas aliadas.

(1). N: Empleo Nacional.

(2). E: Empleo Extranjero.

Fuente: OECD (1994). Elaboración propia.

En general, los trabajadores extranjeros de países comunitarios tienden a concentrarse en los servicios avanzados mucho más que los nacionales. La vinculación de estos trabajadores hacia las actividades terciarias se aprecia particularmente en Francia en el sector de los transportes y comunicaciones; en Bélgica en el comercio y en las instituciones bancarias; y en el Reino Unido en las mismas instituciones bancarias y en la administración pública. Pero el mejor ejemplo se encuentra en Alemania y Luxemburgo, donde el crecimiento del empleo entre los trabajadores extranjeros es más alto en casi todas las actividades de los servicios que entre los nacionales (OECD, 1994).

c) Migraciones internacionales de población retirada

Las Migraciones Internacionales de población Retirada (en adelante MIR) son uno de los rasgos más significativos del mapa social y demográfico de la nueva Europa (CHAMPION y KING, 1993). En el contexto de la descentralización al que hemos aludido anteriormente es donde tenemos que encuadrar estos movimientos de población 
retirada que han surgido en las dos últimas décadas, con origen fundamentalmente en centros metropolitanos densamente poblados, y con destino en pequeñas ciudades o zonas rurales/costeras de países del Mediterráneo. Como vemos, este proceso de suburbanización se desarrolla en un polo totalmente opuesto al de otros grupos de edad más jóvenes en que, por el contrario, se dirigen hacia las grandes ciudades y centros metropolitanos en busca de posibilidades económicas y profesionales (CHAMPION, 1995).

Se trata de un fenómeno con grandes implicaciones sociales y económicas dadas las redistribuciones de gastos sociales, en cuidados y en salud que acarrean, y sobre el que queda mucho por conocer debido a la parquedad de información estadística (WILLIAMS, KING y WARNES, 1997). Su interés se centra en el impacto económico que estos grupos de retirados tienen en los países de destino, debido a que se trata de un grupo de población que ha culminado, o casi, su ciclo productivo (profesionalmente hablando) y que cuentan con cantidades de dinero importantes que normalmente son transferidas a los países de acogida. Pero tampoco hay que olvidar el impacto cultural y social que estos grupos tienen en los países receptores, donde generalmente habitan en enclaves formados en la mayoría de los casos por población extranjera.

Haciendo un breve repaso de la bibliografía existente sobre el tema, la mayoría de ella ha tratado únicamente los desplazamientos intra-nacionales de retirados. Por ejemplo, CRIBIER (1982) y CRIBIER y KYCH (1992) provén un excelente análisis de las MIR en Francia, mientras que CRIBIER y KYCH (1993) comparan las MIR con origen en Londres y en París. En ambos casos, las motivaciones para emigrar las constituye los deseos de paz y tranquilidad, y la atracción de los medios rurales y 1 a vida relajada, unido al retorno a las zonas de origen, el desplazamiento a zonas donde habitan familiares próximos (hijos o nietos), o el interés por zonas turísticas y soleadas bien dotadas de infraestructuras y servicios (Côte d'Azur). En otros estudios como los realizados en el Reino Unido por WARNES (1993), y por OBERG y ot. (1993) en Suecia, las tendencias detectadas fueron similares.

Una cuestión importante es si las características analizadas para los desplazamientos intranacionales pueden ser aplicadas a estas migraciones internacionales a zonas mediterráneas. Muchas veces pueden darse similaridades obvias entre ambos tipos como son las motivaciones para el desplazamiento (para estar cerca de la familia o en forma de retorno a los lugares de origen), en la elección del destino (zonas costeras o rurales), aunque no siempre ocurre así. Cada vez más, retirados de países del norte europeo, sin que se establezca ninguna relación familiar, y tras conocer el país sólo de haber pasado en él algunas vacaciones, deciden fijar su residencia a orillas del Mediterráneo sin más atracción que la que puede producir su tranquilidad y su clima. No olvidemos tampoco los muchos retirados que desean ponerse al resguardo de los fuertes regímenes recaudatorios de sus países de origen 
transfiriendo todos sus ingresos a países con unos regímenes de impuestos menos opresores. El hecho, por tanto, de que las MIR se lleven a cabo a través de las fronteras, y que sólo excepcionalmente impliquen el contacto entre familias, hace que las consecuencias de este tipo de migraciones sean distintas a las producidas dentro del mismo país.

A la hora de tomar la decisión de emigrar, intervienen en ella muchos factores tanto de índole económico, como sociológico, cultural o climático. Si tuviéramos que resumir esos factores, se podrían destacar tres como los principales. (i) El primero tiene que ver con los cambios demográficos producidos en Europa en las últimas décadas. Pero si bien el potencial para las MIR ha aumentado con el rápido envejecimiento de la población noreuropea, eso no justifica por sí solo el crecimiento de este tipo de movilidad. Lo normal sería pensar que no siempre todos los retirados están dispuestos a emigrar a otro país.

(ii) El segundo factor que explicaría este crecimiento son determinados cambios en la economía europea. El aumento de la renta per cápita en este grupo concreto de población hace aumentar, en principio, su capacidad económica. Junto a ello, el crecimiento del desempleo en Europa desde los años 70 ha llevado a muchos trabajadores al retiro cada vez más jóvenes, obteniendo sustanciales indemnizaciones por jubilación. A esos mismos cambios económicos se podrían atribuir el aumento sustancial de los precios de las viviendas en la década de los 80 en los países de origen, y el menor precio de las viviendas en los de destino con el ahorro sustancial que en $\mathbf{m}$ país mediterráneo puede suponer debido a su clima (por el ahorro en calefacción). Aunque los costes diferenciales de vida entre los países del norte y del sur de Europa se han aproximado bastante en las últimas décadas, el clima en primer lugar, como las diferentes culturas del sur, el paisaje y los distintos modos de vida, son las razones principales esgrimidas por más de la mitad de los extranjeros europeos con residencia en Alicante (VALERO, 1992). Esas consideraciones sobre la "calidad de vida", entendida como un conjunto de aspectos físicos y sociales, son las que más pesan a la hora de decidir la emigración. Tales consideraciones se traducen en una percepción global referida a las zonas de destino en la que influyen factores como la poca criminalidad y escasa congestión y polución, las buenas condiciones climáticas, o el creciente acceso a servicios de alimentación, transporte y sanitarios (CHAMPION, 1995).

(iii) El tercer factor para entender este tipo de emigración son los hábitos de movilidad que la población europea está adquiriendo, en parte debido a la mayor facilidad hoy en día para poder vivir y trabajar en otros países. Los cambios de residencia debido al trabajo traspasan cada vez más las fronteras del propio país, lo cual se traduce en mayor experiencia de vivir y trabajar en el extranjero. Ligado a ello, como se verá en el siguiente apartado, el crecimiento del turismo internacional ha 
hecho también aumentar la experiencia y el conocimiento de otros países, aunque sea de una manera superficial y rápida como es con los paquetes de vacaciones (WILLIAMS y SHAW, 1988). De esa forma, repetidas vacaciones suelen convertirse en un primer paso para migraciones posteriores estacionales o permanentes, o como una vía para la adquisición de una segunda residencia en otro país.

Determinar el volumen exacto que las MIR representan es una tarea díficil debido a los distintos sistemas de recolección de datos que se usan entre todos los países europeos, y no siempre coincidentes en el tiempo. Pero pese a eso, parece estar claro que los principales flujos proceden de países del norte, y se dirigen hacia los del sur. Según datos de la Oficina de Publicaciones de las Comunidades Europeas, casi la mitad de los residentes extranjeros europeos de Italia procedían de países del norte de Europa, en el caso de Grecia más de la mitad, en Portugal un 70\% y en España casi un 75\% (Tabla 4). En los cuatro países, Alemania, Reino Unido y Francia son los tres principales emisores. Salvo en Italia, donde su porcentaje es más bajo $(8,7 \%)$, los británicos en Grecia $(19,4 \%)$, Portugal $(26,9 \%)$ y España $(27,1 \%)$ suponen la mayoría de los extranjeros europeos.

Las investigaciones sobre inmigrantes retirados en zonas mediterráneas son todavía escasas, pero entre las realizadas, se puede afirmar que los flujos fundamentales tienen lugar en sentido norte-sur (WARNES, 1994). Entre los estudios realizados sobre las consecuencias tanto para los emigrantes como para las comunidades de recepción cabe destacar el de JURDAO y SÁNCHEZ (1990), en donde se trata el impacto cultural y económico de los emigrantes noreuropeos (incluyendo los retirados) en uno de los municipios más densamente "colonizados" por residentes extranjeros como es Mijas, en la Costa del Sol. Según VALENZUELA (1991), más de la mitad de los extranjeros propietarios de residencias secundarias son británicos o alemanes, y su número se podía estimar en más de 500.000 entre la Costa del Sol y la Costa Blanca. Trabajos que estudian también este tipo de migraciones son los de BULLER y HOGGART (1994), y HOGGART y BULLER (1995). Referidos en ambos casos a la compra de residencias secundarias por parte de británicos en Francia, los autores identifican al grupo concreto de los retirados, cuyo número ha pasado en la década de los 80 de los 2.000 en 1987 a 14.000 en 1989. También para Francia, el trabajo de BAROU y PRADO (1995) analiza el impacto de los británcios en regiones atractivas para esos ciudadanos como son la Bretaña, Normandía, el Périgord y la Ardèche. Pero aparte de estos y otros pocos trabajos llevados a cabo principalmente en zonas italianas, el número de investigaciones no es mucho más numeroso.

Se trata de un tipo de migración difícil de definir, por cuanto el límite entre lo que constituye propiamente una "migración" más o menos estacional o de más larga duración, y las muchas formas de hacer turismo, es muy impreciso. Dentro de todo este continuo que suponen estas migraciones se pueden destacar las de la tercera edad en 
forma de largas estancias vacacionales ligadas fundamentalmente al turismo. E1 crecimiento actual del turismo de tercera edad (estimado hoy en unos 50 millones de desplazamientos por año; ECONOMIST INTELLIGENCE UNIT, 1993) lo convierte en uno de los segmentos con mayor expansión en el mercado, estimándose que el número de personas con más de 55 años que en Europa salen de vacaciones al extranjero ha aumentado aproximadamente un 35\% entre 1990 y 1995. Tal mercado está actualmente dominado por países noreuropeos, destacando por orden de importancia en el origen de la demanda Alemania (32\%), el Reino Unido (15\%) y Francia (9\%) como principales países emisores. Sólo haciendo referencia a británicos que abandonaron su país por $\mathrm{m}$ periodo de un año o más, se estima que desde 1975 han salido de Gran Bretaña un número entre 100.000 y 190.000, no siendo continuo ni pudiéndose detectar en él ninguna tendencia clara. Entre ellos, el porcentaje de retirados ha variado desde los 5.700 anuales entre 1975-80, a los 7.200 entre 1987-92 (WILLIAMS, KING y WARNES, 1997; tomado de OPCS, 1994). En resumen, bien como desplazamientos de corta, o más larga duración, este tipo de movilidad constituye una de las principales características de los movimientos de población intracomunitaria en la actualidad.

\section{d) Migraciones y turismo en zonas del Mediterráneo}

Hasta cierto punto, uno de los factores que ha contribuido al aumento de los desplazamientos de población retirada ha sido la facilidad para viajar, y el conocimiento (previo al desplazamiento más definitivo) de otros países y culturas. Por lo tanto, el turismo en sí es otro de los factores que merecen atención en la explicación no sólo de ese tipo de movilidad, sino de la que él mismo genera. Muchos estudios han analizado, en general, la mutua relación entre la reestruturación económica y la movilidad socioespacial de la población europea a escalas nacional y regional; sin embargo, se ha prestado escasa atención al turismo y al efecto que tiene en las migraciones.

Como ha escrito R. King, la reestructuración económica ha teniendo efectos variados en los diferentes grupos de emigrantes y, concretamente, gran influencia en el sector turístico en relación con la movilidad de la población europea, en particular desde países del norte de Europa a los del arco mediterráneo (KING, 1997).

Pero antes de analizar la movilidad generada por el turismo, conviene hacer una precisión de carácter terminológico. 
Tabla 4.-Extranjeros de países europeos residentes en países del sur de Europa, 1993.

\begin{tabular}{|c|c|c|c|c|c|c|c|c|}
\hline \multirow[b]{2}{*}{ Pais(es) de origen } & \multicolumn{2}{|c|}{ ITALIA } & \multicolumn{2}{|c|}{ GRECIA } & \multicolumn{2}{|c|}{ PORTUGAL } & \multicolumn{2}{|c|}{ ESPAÑA } \\
\hline & $N^{\prime \prime}(1)$ & $\%(2)$ & $N^{\prime \prime \prime}$ & $\%$ & $N^{\prime \prime}$ & $\%$ & $\mathrm{~N}^{\prime \prime}$ & $\%$ \\
\hline Bélgica & 4,6 & 1,4 & 1,7 & 1,6 & 1,1 & 3,1 & 7,2 & 3,6 \\
\hline Dinamarca & 2,4 & 0,7 & 1,6 & 1,5 & 0,5 & 1,4 & 4,0 & 2,0 \\
\hline Alemania & 39,5 & 12,1 & 14,1 & 13,2 & 5,4 & 15,5 & 30,5 & 15,4 \\
\hline Francia & 25,4 & 7,7 & 8,0 & $7, \overline{5}$ & 3,7 & 10,6 & 22,6 & 11,4 \\
\hline Países Bajos & 7,0 & 2,1 & 3,7 & 3,4 & 2,0 & 5,7 & 10,5 & 5,3 \\
\hline Reino Unido & 28,4 & 8,7 & 20,7 & 19,4 & 9,3 & 26,9 & 53,4 & 27,1 \\
\hline Irlanda & 2,5 & 0,7 & 0,7 & 0,6 & 0,2 & 0,5 & 2,1 & 1,1 \\
\hline Suiza & 18,2 & 5,5 & 1,9 & 1,7 & 0,7 & 2,0 & 5,6 & 2,8 \\
\hline Austria & 8,3 & 2,5 & 1,9 & 1,7 & 0,3 & 0,8 & 1,5 & 0,7 \\
\hline Noruega & 0,9 & 0,3 & 0,8 & 0,7 & 0,3 & 0,8 & 2,4 & 1,2 \\
\hline Suecia & 3,2 & 1,0 & 2,3 & 2,1 & 0,7 & 2,0 & 5,3 & 2,7 \\
\hline Otros Norte Europa & 2,0 & 0,6 & 1,2 & 1,1 & 0,2 & 0,5 & 2,1 & 1,1 \\
\hline Total Norte Europa & 142,4 & $(43,6)$ & 58,6 & $(54,8)$ & 24,4 & $(70,3)$ & 147,2 & $(74,6)$ \\
\hline Total UE (3) & 160,3 & $(49,1)$ & 64,7 & $(60,6)$ & 32,7 & $(94,2)$ & 181,8 & $(92,1)$ \\
\hline TOTAL EUROPA & 326,4 & 100,0 & 106,8 & 100,0 & 34,7 & 100,0 & 197,3 & 100,0 \\
\hline
\end{tabular}

(1) En miles (1000).

(2) Porcentaje de europeos de cada nacionalidad en el conjunto del país.

(3) Incluidos para cada país los otros residentes de países del sur (Italia, Grecia, Portugal, o España).

Fuente: EUROSTAT (1995). Elaboración propia. 
Los desplazamientos turísticos no deberían incluirse en la misma categoría que las migraciones propiamente dichas. Su inclusión, sin embargo, como un tipo de migraciones (de corta duración) responde a una razón de tipo conceptual y terminológica, aun sabiendo que las características entre ambos tipos de movilidad son notables. De la misma forma que es difícil precisar qué se entiende por migración de población retirada, y sus límites (a veces puede tratarse sólo de una migración estacional e intermitente, lo cual supone una estancia turística de larga duración), los largos desplazamientos turísticos se pueden considerar como migraciones de corta duración. De ahí que en este análisis de las migraciones y movilidad incluyamos el turismo como una nueva modalidad de migración, sobre todo por el enorme volumen de población que implica, y por esa imprecisión que supone determinar dónde acaba un desplazamiento turístico y empieza una migración.

Hecha esa precisión, lo primero que hay que resaltar al referirnos al sector turístico y al turismo en Europa es su rápida expansión en las últimas décadas. En toda Europa occidental se llegó a concentrar en 1984 el 68\% de todo el turismo internacional mundial (WILLIAMS y SHAW, 1988), y según la ONU, la zona mediterránea es la más frecuentada turísticamente a nivel mundial, con más de 100 millones de visitantes en 1985, ó 113 millones de paquetes turísticos vendidos para vacaciones cada año (PERRY y ASHTON, 1994). En respuesta a tal demanda, los países del sur europeo han desarrollado una enorme industria con España a la cabeza en cuanto a número anual de visitantes: si en 1960 fueron 6 millones, en el año 1994 se sobrepasaron los 61 millones (S.G.T., 1995). Tal especialización turística genera millones de desplazamientos, la mayoría de ellos con origen en países comunitarios. Tal y como se aprecia en la Tabla 5 para el caso español, sólo cuatro países europeos generan casi el 70\% del turismo extranjero. Junto a España, países como Francia, Italia, Grecia, o más recientemente Turquía, han seguido igualmente el tren del desarrollo, convirtiéndose en destinos masivos por parte de la creciente demanda europea. De ahí que, hoy por hoy, los principales flujos turísticos en los países comunitarios se lleven a cabo en sentido nortesur, lo cual implica una gran concentración tanto en la emisión como en la recepción turística.

De alguna forma, este crecimiento de las actividades turísticas ha supuesto uno de los desarrollos económicos y sociales más importantes en Europa en las últimas décadas, representando un símbolo de la reconstrucción de Europa occidental (URRY, 1996). En ese sentido MARCHENA (1995) ha resaltado también el papel que el turismo ha tenido y está teniendo en la construcción y creación de la unidad europea, y el hecho de que la internacionalización de los flujos económicos junto con el concepto de una Europa unida ofrecen cierta estabilidad para su práctica desde el punto de vista socioeconómico o político. Según MARCHENA (1995), durante las décadas de los 80 y los 90 se han producido cambios radicales en la actitud del turismo, que han producido la expansión y crecimiento de la actividad en las zonas del Mediterráneo. En primer 
lugar, la integración del producto turístico en el mercado de masas ha supuesto que el turismo haya llegado a la gran masa, en función de los diferentes estratos sociales y económicos. Sin duda, la mayor disponibilidad de tiempo y también económica, son factores que han contribuido a la expansión del sector. Pero este crecimiento hay que contextualizarlo en la globalización de la economía mundial y en particular de la europea, y explicar así la internacionalización del sector. En concreto, el desarrollo de los tour operators ha permitido que miles de turistas puedan desplazarse con sus paquetes de vacaciones a precios cada vez más asequibles, convirtiéndose de esa manera el paquete turístico en algo popular y accesible a las pequeñas economías de la mayoría de trabajadores de los países del norte de Europa.

Esa internacionalización del turismo comenzó con mayor fuerza en países como Gran Bretaña (PEARCE, 1995; URRY, 1996) debido en parte al temprano y rápido desarrollo de los touroperadores. El número de ellos era de 679 en el año 1988 (WILLIAMS, 1988), y el total de paquetes de vacaciones vendidos ha pasado de los 5 millones en 1960 a los 10 millones vendidos en 1992 (PERRY y ASHTON, 1994). En 1 a actualidad, cerca de 30 millones de personas usan cada año tour-operadores británicos para desplazarse a zonas del Mediterráneo, lo que constituye un $65 \%$ de todos los destinos (BRACKENBURY, 1997). Tal éxito en la expansión de la industria de los paquetes de vacaciones se debe al considerable grado de concentracion y de integración horizontal y vertical en el sector turístico. El resultado ha sido la expansión de todo un sector empresarial dominado por las alianzas entre compañías y touroperadores, sacando rápida ventaja de los avances en las comunicaciones aéreas y de los sistemas de reservas por ordenador (REYNOLDS, 1989). Pero la expansión de este turismo masivo, a su vez, ha traido consigo el crecimiento de un tipo de movilidad laboral. En ese sentido, GORDON (1991) hace referencia a la importancia que está teniendo el proceso de desindustrialización y en general la reestructuración económica en la creación de nuevos empleos en el sector terciario, implicando todo ello nuevas oportunidades laborales para emigrantes extranjeros, especialmente en los servicios turísticos.

Todo ello ha permitido el aumento de la movilidad geográfica de muchos inmigrantes, si bien eso es algo sobre lo que no se dispone de poca información estadística. Eso se traduce, por un lado, en el aumento de negocios turísticos en manos de inmigrantes, favorecido por el no requerimiento de específicas cualificaciones y por la cada vez mayor facilidad administrativa entre los comunitarios para crear este tipo de empresas. Por otro, nos referimos a la creación de empleos turísticos en los lugares de destino, generalmente ocupados por trabajadores extranjeros, como es el caso de los 
Tabla 5.- Turistas extranjeros en España, 1994.

\begin{tabular}{lrc}
\hline Origen de los turistas & Total & $\%$ \\
\hline Francia & 13.696 .012 & 22,3 \\
Portugal & 10.249 .070 & 16,7 \\
Alemania & 9.678 .673 & 15,8 \\
Reino Unido & 9.170 .041 & 14,9 \\
Italia & 2.818 .988 & 4,6 \\
Holanda & 2.271 .429 & 3,7 \\
Bélgica & 2.076 .267 & 3,4 \\
Suiza & 1.990 .268 & 3,2 \\
Resto Europa & 2.606 .208 & 4,2 \\
TOTAL EUROPA & 54.556 .956 & 88,8 \\
Estados Unidos y Canadá & 277.497 & 1,9 \\
Argentina & 224.832 & 0,4 \\
Brasil & 227.843 & 0,4 \\
Resto de América & 297.277 & 0,5 \\
RESTO MUNDO & 4.945 .629 & 8,0 \\
\hline
\end{tabular}

Fuente: Movimientos Turisticos en Fronteras. S.G.T., tomado de S.G.T. (1995).

trabajadores de touroperadores ${ }^{2}$. La expansión de este tipo de empleos temporales hay que relacionarla con la alteración de la naturaleza de la demanda laboral en la era post-Fordista. En concreto, uno de los cambios que ha originado la polarización tanto espacial como temporal de la demanda de servicios turísticos ha sido la del aumento de trabajo de tipo casual, temporal, y mucho más flexible (KING, 1997). Los

2 En este sentido la UE ha adoptado una serie de directivas que permiten a todos los ciudadanos trabajar, bien como empleados en el sector turístico, o con negocios propios, en cualquier país de la Unión. La directiva 75/368/EEC regula la libertad en el establecimiento de personas que ejercen las profesiones de guía turístico y organizador de tours en otro país (tour manager), bien trabajando como organizadores de tours-circuitospaquetes de vacaciones, o como acompañante de grupos de turistas pero establecidos en un distinto país al suyo; por el contrario, esa libertad de establecimiento no está contemplada para los guías que sólo acompañan a grupos de turistas, y se dedican a dar información y explicaciones de los lugares visitados (EUROPEAN COMMISSION, 1997). 
touroperadores cuentan en los países mediterráneos en donde trabajan con numeroso personal que se ocupa de las tareas de organización y coordinación principalmente del alojamiento $y$ de las actividades ofertadas a sus turistas. Generalmente son profesionales que residen en estos países durante la temporada turística, y que trabajan como animadores de grupo, representantes de ventas, relaciones públicas, administrativos, etc.

Ante tal situación, y con el fin de prever futuros flujos y movimientos ligados a 1 turismo en zonas del Mediterráneo, parece evidente que el modelo del turismo masivo es algo que no vaya a desaparecer rápidamente, en la medida que abastece la demanda de millones de turistas anualmente. De hecho, y como ha escrito MARCHENA (1995, p. 30), "el modelo turístico del Mediterráneo todavía está ligado a la idea de sol y arena para las vacaciones de las masas"; es probable, por tanto, que el turismo siga constituyendo uno de los principales factores de movilidad en el área.

\section{CONCLUSIONES}

La libre circulación de personas está siendo una de las cuestiones más delicadas de la puesta en marcha del mercado interior europeo, menos por razones económicas, pero sí por las de tipo social, cultural y psicológicas. La movilidad de la población comunitaria es algo muy complejo, ligado tanto a factores demográficos y económicos, como a otros factores más específicos relacionados con la heterogeneidad de la Unión Europea y con la dificultad de una completa abertura al espacio comunitario (SIMON, 1992). Desde el punto de vista económico, tal y como hemos considerado, parece que hay ciertos indicios para que la movilidad entre países de la U.E. vaya en aumento. La lógica hace suponer que, a nivel de corporaciones individuales, la tendencia del crecimiento de la movilidad de los trabajadores cualificados, es limitada, aunque lo incierto es saber cuándo disminuirá. Por un lado, ciertas empresas están adoptando estrategias para reducir esa movilidad de sus trabajadores, bien mediante fusiones con otras, o bien haciendo uso de los servicios especializados para empresas, trabajo desde el domicilio, etc. Otras, sin embargo, están todavía internacionalizando sus actividades, en cuyos casos la movilidad de trabajadores se seguirá produciendo. E1 cómo evolucione este tipo de migración dependerá, por tanto, de estas dos tendencias.

En relación con las diferencias norte-sur, es bastante poco probable que en un futuro próximo determinadas fuerzas económicas dejen en una posición de desventaja a 1 actual "centro" europeo, constituido espacialmente en torno al eje o triángulo LondresParís-Frankfurt. Lo que parece cierto es que, aparte de ese "centro" que seguirá actuando como polo de atracción, el eje Mediterráneo va a seguir configurándose como un eje de desarrollo de alta tecnología industrial y de servicios, incluidos los turísticos. 
Por otro lado, y combinado con todo ello, la efectividad de las diferentes políticas comunitarias (de desarrollo regional, rural, industrial, turístico, etc.) es algo que todavía está por demostrar de cara a la futura prevención o agravamiento de los desequilibrios internos entre las regiones más y menos favorecidas, y de cara a lo que eso supondría en cuanto a desplazamientos de población. Aunque hablando de desequilibrios, la puesta en marcha de la moneda única es probable que introduzca importantes desigualdades en la capacidad adquisitiva de los emigrantes dependiendo de los distintos países comunitarios, lo cual afectará fuertemente a los desplazamientos de población.

En cuanto a las perspectivas del sector turístico y la influencia que eso puede tener en la variación del número de desplazamientos, no parece que vaya a haber grandes cambios. Tal y como señalaron recientemente miembros de la Federación Internacional de Tour-Operadores $y$ de la Asociación de Tour-Operadores Independientes (véase la referencia de BRACKENBURY, 1997), el turismo en zonas del Mediterráneo servido por tour-operadores está suficientemente consolidado, y 1 a demanda de destinos en el área está asegurada pese a la incorporación de otros nuevos, más lejanos y exóticos. Referido no sólo a los desplazamientos turísticos, sino a todos los tipos de movilidad que hemos analizado, parece que predominarán los de corta duración (de pocos días, meses o años, dependiendo del tipo), sobre los de larga duración. De aquí que el concepto de "movilidad" se imponga al de "migración", por cuanto este último puede asociarse a desplazamientos de mayor duración y carácter más definitivo.

Sin embargo, hay una serie de factores de tipo cultural y social, menos "visibles" y "materiales", que son los que están restringiendo y limitando la movilidad. En la práctica, quedan diversos aspectos prácticos por solucionar, emergentes de la diversidad y heterogeneidad a la que la unión, y pretendida integración de quince países, conduce, y que hoy por hoy restringen fuertemente la movilidad intracomunitaria. Entre todos, los factores que surgen de las inevitables diferencias y diversidades culturales entre los países comunitarios. La multiplicidad lingüística, de costumbres y prácticas culturales de cada país, constituye importantes barreras para la comunicación, y en consecuencia, para la movilidad. Aunque a nivel estrictamente profesional todavía quedan obstáculos que vencer como las barreras de pertenecer a cuerpos profesionales determinados para ejercer la profesión en países concretos, o el hecho de que determinadas asociaciones profesionales exigan cualificaciones reconocidas en el propio país, hay otros muchos de tipo social (pérdida de ciertos derechos asistenciales y de protección, problemas de incompatibilidades y no reconocimientos administrativos por parte del país de acogida, etc.), que limitan enormemente los desplazamientos. Son sólo algunos ejemplos de cómo determinados factores culturales y sociales pueden actuar como un freno en la llamada "Europa sin fronteras" en el proceso de la emigración, y de cómo la apertura del Mercado Único 
Europeo ha tenido un escaso efecto en la reducción "mental" de las fronteras culturales y sociales entre los países comunitarios. En definitiva, si la nueva construcción europea ha logrado avances significativos en cuando a la supresión de las barreras físicas y económicas para la movilidad de la población, todavía queda bastante camino por recorrer en cuanto a la supresión de las otras: las "invisibles".

\section{BIBLIOGRAFÍA}

BAROU, J. y PRADO, P. (1995): Les Anglais dans nos Campagnes. Ed. L' Harmattan, Paris.

BRACKENBURY, M. (1997): Tourism in the Mediterranean: Challenges and Opportunities, Actas de la Conferencia, Westminster University, Londres, 2 de Diciembre de 1997.

BULLER, H. y HOGGART, K. (1994): International Counterurbanization: British Migrants in Rural France. Ed. Avebury, Aldershot.

CHAMPION, A.G. e ILLERIS, S. (1990): Population redistribution trends in western Europe. En: HEBBERT, M. \& HANSEN, J.C. (Eds.):Unfamiliar territory: the reshaping of European geography. 236-253. Avebury. Aldershot.

CHAMPION, T. (1995): Internal migration, counterurbanization and changing population distribution. En: HALL, R. y WHITE, P. (Eds.): Europe's Population. Towuards the next century. 99-129. UCL \& Press. London.

y KING, R. (1993): New Trends in International Migration in Europe. Geographical Vieropoint. 21, 45-56.

CRIBIER, F. (1982): Aspects of Retirement Migration from Paris: An Essay in Social and Cultural Geography. En: WARNES, A.M. (Ed.): Geographical Perspectives on the Elderly. 111-137. Wiley. London.

- y KYCH, A. (1992): La migracion de retraite des Parisiens: une analyse de la propension au départ. Population. 47, 677-718.

y KYCH, A. (1993): A Comparison of Retirement Migration from Paris and London. Environment and Planning A. 25, 1399-1420.

ECONOMIST INTELLIGENCE UNIT (1993): Europe's Senior Travel Market. Travel and Tourism Analyst. 4, 37-56.

EUROPEAN COMMISSION (1996): Marie Curie Research Training Grants. Information Package 1996. Ed. European Commission. Brussels.

(1997): Supporting Culture, Sport and Tourism, Ed. Representación de la Comisión Europea en el Reino Unido. London.

EUROSTAT (1995): Migration Statistics. 1995. Ed. Eurostat. Brussels. 
FINDLAY, A.M. (1995): The future of skill exhanges withing the European Union. En: HALL, R. \& WHITE, P. (Eds.): Europe's Population. Touords the next century. 130-141. UCL \& Press. London.

GORDON, I. (1991): The Impact of Economic Change on Minorities and Migrants in Westem Europe. University of Reading. Department of Geography. Discussion Paper 2.

HOGGART, K. y BULLER, H. (1995): Retired British Home Owners in Rural France. Ageing and Society. 15, 325-353.

JURDAO, F. y SÁNCHEZ, M. (1990): España, Asilo de Europa. Ed. Planeta. Barcelona.

KAMEOKA, Y. (1996): The Internationalization of Higher Education. OECD Observer. 202, October/November, 34-36.

KING, R. (1993): The Nerv Geograply of European Migrations. Ed. Belhaven. London and New York.

(1997): Restructuring and Socio-Spatial Mobility in Europe: The role of International Migrants. En: BLOTEVOGEL, H.H. y FIELDING, A.J. (Eds.): People, Jobs and Mobility in the New Etrope. 91-119. Wiley. London.

MAJAVA, A. y PENTTINEN, M. (1991): Sopemi-Finland 1990. Ed. OECD. Paris.

MARCHENA, M. J. (1995): New tourism trends and the future of Mediterranean Europe. Tijdschrift voor Economische en Sociale Geografie. 86, 1, 21-31.

MUUS, P. (1991): Sopemi-Netherlands 1990. Ed. OECD. Paris.

OBERG, S.; SCHEELE, S. y SUNDSTRÖM, G. (1993): Migration among the Elderly: The Stockholm Case. Espaces, Populations, Sociétés. 3, 503-514.

OECD (1994): Trends in International Migration. Annual Report. SOPEMI, 1993. Ed. OECD. Paris.

OPCS (1994): International Migration (1992). Ed. HMSO, Series MN, 19. London.

PEARCE, D. G. (1995): An integrated approach to international tourist flows. Tourism Today: A geographical analysis. 82-94. Longman Scientific \& Technical. London.

PÉREZ, S. (1996): "Los españoles obtuvieron en 1995 el mayor número de becas de investigación de la UE", en El País, viernes 5 de enero de 1996, p. 25.

PERRY, A. y ASHTON, S. (1994): Recent Developments in the UK's Outbound Package Tourism Market. Geograpliy. 79, 313-321.

REYNOLDS, H. (1989): The 100 Best Companies to Work for in the UK. Ed. Fontana / Collins. London.

SALT, J. (1992): Migration Processes among the Highly Skilled in Europe. International Migration Review. 26, 2, 484-505. 
S.G.T. (SECRETARÍA GENERAL DE TURISMO) (1995): Nota de Coynntura Turística. Ed. S.G.T., Enero. Madrid.

SIMON, G. (1992): Une Europe Communautaire de moins en moins mobile?. Revue Européenne des Migrations Internationales. 8, ( $\mathrm{n}$ " hors de serie), 67-87.

(1993): Internal Migration and Mobility. En: NOIN, D. y WOODS, R. (Eds.): The Changing Population of Europe. 170-184. Blackwell. Oxford-Cambridge.

URRY, J. (1996): The changing economics of the tourism industry. En: APOSTOLOPOULOS, $X$; LEIVADI, S. y YIANNAKIS, A. (Eds.): The Sociology of Tourism. 193-218. Routledge. New York and London.

VALENZUELA, M. (1991): "The Phenomenon of Mass Tourism. En: WILLIAMS, A.M. y SHAW, G. (Eds.): Tourism and Econonic Development: Western European Experiences. 40-60. Belhaven Press. London.

VALERO ESCANDELL, J.R. (1992): La Inmigración Extranjera en Alicante. Ed. Instituto de Cultura Juan Gil-Albert. Alicante.

WARNES, A.M. (1993): Demographic ageing: Trends and Policy Response. En: NOIN, D. y WOODS, R. (Eds.): The Changing Population of Europe. 82-99. Blackwell. Oxford.

(1994): Permanent and Seasonal International Retirement Migration: The Prospects for Europe. Netherlands Geographical Studies. 173, 69-81.

WERNER, H. (1991): Migration Movements in the Perspective of the Single European Market. International Conference on Migration. Ed. OECD/Republic of Italy. Rome.

WILLIAMS, A.M.; KING, R. y WARNES, T. (1997): A Place in the sun: International Retirement migration from northern to southern Europe. European Urban and Regional Studies. 4, 2, 115 134.

WILLIAMS, A. y SHAW, G. (1988): Tourism and development: introduction. En: WILLIAMS, A. y SHAW, G. (Eds.): Tourism and Economic Development. 1-11. Belhaven Press. London. 